\title{
Poly(lactic-co-glycolic acid) Bone Scaffolds with Inverted Colloidal Crystal Geometry
}

\author{
MEGHAN J. CUDDIHY, M.S., ${ }^{1}$ and NICHOLAS A. KOTOV, Ph.D. ${ }^{1-3}$
}

\begin{abstract}
Controllability of scaffold architecture is essential to meet specific criteria for bone tissue engineering implants, including adequate porosity, interconnectivity, and mechanical properties to promote bone growth. Many current scaffold manufacturing techniques induce random porosity in bulk materials, requiring high porosities ( $>95 \%$ ) to guarantee complete interconnectivity, but the high porosity sacrifices mechanical properties. Additionally, the stochastic arrangement of pores causes scaffold-to-scaffold variation. Here, we introduce a biodegradable poly(lactic-co-glycolic acid) (PLGA) scaffold with an inverted colloidal crystal (ICC) structure that provides a highly ordered arrangement of identical spherical cavities. Colloidal crystals (CCs) were constructed with soda lime beads of 100-, 200-, and 330- $\mu \mathrm{m}$ diameters. After the CCs were annealed, they were infiltrated with 85:15 PLGA. The method of construction and highly ordered structure allowed for ease of control over cavity and interconnecting channel diameters and for full interconnectivity at lower porosities. The scaffolds demonstrated high mechanical properties for PLGA alone ( $>50 \mathrm{MPa}$ ), in vitro biocompatibility, and maintenance of osteoblast phenotype, making them promising for a highly controllable bone tissue engineering scaffold.
\end{abstract}

\section{INTRODUCTION}

B ONE TISSUE ENGINEERING aims to replace traditional autograft and allograft repair strategies with the implantation of mechanically functional biodegradable constructs that support the wound site and hasten the body's natural repair mechanism until bone has filled the defect. ${ }^{1,2} \mathrm{~A}$ number of structural criteria for implantable scaffolds are agreed upon, including sufficient porosity, interconnectivity, and mechanical properties. Porosity and complete interconnectivity throughout a scaffold are necessary to promote uniform cell loading and migration, eventual tissue and vasculature ingrowth, and sufficient nutrient diffusion and interstitial fluid and blood flow. ${ }^{3,4}$ The ideal bone tissue engineering scaffold should have a precisely designed structure that provides complete interconnectivity of pores and maximum load bearing capacity. ${ }^{5}$
Traditional scaffold fabrication techniques, such as particulate leaching, gas foaming, emulsion freeze-drying, electrospinning, and thermally induced phase separation, are based upon bulk material alteration that yields a random pore structure. ${ }^{6-10}$ The random pore distribution risks insufficient scaffold interconnectivity and permeability; consequently, scaffold porosities often approach $95 \%$ to ensure complete interconnectivity throughout the scaffold. Such high porosities present a dilemma in bone tissue engineering, where greater porosity enhances interconnectivity but denies the scaffold sufficient mechanical strength to support the load of the body until tissue regeneration is complete. Therefore, there is a need for structural engineering of bone scaffolds, providing sufficient control over threedimensional (3D) geometry. ${ }^{11,12}$ A systematic approach to this problem would be the preparation of well-ordered porous materials with tunable geometry. Computational

Departments of ${ }^{1}$ Chemical Engineering, ${ }^{2}$ Materials Science and Engineering, and ${ }^{3}$ Biomedical Engineering, University of Michigan, Ann Arbor, Michigan. 
image-based design and fabrication processes, such as solid free-form (SFF) fabrication, provide precise control over scaffold architecture, including interconnectivity and geometry, but are limited in useable materials and have a minimum feature size of $100 \mu \mathrm{m} .^{4,11,13,14}$ A method of producing scaffolds with highly controlled architectural properties on multiple scales would greatly advance scaffold design for bone tissue engineering.

Inverted colloidal crystal (ICC) scaffolds have emerged recently as a highly organized 3D environment for cell growth. ${ }^{15-20}$ ICC scaffolds possess a hexagonal closepacked (HCP) geometry, which can be described as closely packed spherical cavities arranged in a hexagonal crystal lattice. The HCP arrangement offers a uniform cellular environment for differentiation and growth, as well as an ideal setting for understanding the role of cell-to-cell and cell-toscaffold interactions, and for computer modeling of scaffold properties. ${ }^{18}$ Additionally, HCP geometry guarantees interconnectivity between all neighboring spherical cavities, and the maximum theoretical porosity for HCP structures is $74 \%$. The high interconnectivity and low porosity of hydrogel HCP structures are 10 to 1,000 times stiffer than predicted for bulk material alteration techniques in polyethylene glycol hydrogel scaffolds. ${ }^{20}$ ICC structures can be prepared from any bulk material, as long as a suitable pair of solvents is found: one to dissolve the mold without disturbing the bulk material and vice versa. Ideally, specific design criteria, such as mechanical properties, degradation rate, and growth factor release rate, can be met by adjusting scaffold material and the size of the spheres that compose the mold.

Recently, our group has produced ICC scaffolds composed of sol-gel and polyacrylamide hydrogel. ${ }^{15-19}$ Several cell types were shown to attach to and proliferate on these structures, including human hepatic, bone marrow stromal, thymic epithelial, and monocyte cells and mouse fibroblastic and T-cells. The challenges related to cell attachment typical for hydrogels were solved using layer-by-layer coating of their surfaces. ${ }^{16,21}$ The ICC structure offers some obvious advantages for bone tissue engineering such as a high degree of structural control, invariably complete interconnectivity of cavities, 3D geometry resembling the morphology of trabecular bone, and the possibility of achieving high mechanical strength. Other tangible advantages, which might come into play in future research, are the convenience for tissue development simulation and incorporation of osteoconductive and osteoinductive nanocolloids. From a fundamental point of view, the simplicity of ICC preparation and the high degree of structural control provide excellent opportunities for understanding and manipulating cell culture on the scaffold.

Here, we introduce ICC scaffolds from a biodegradable polymer often used in constructing bone implants. The key parameters examined here are the ability to create highly ordered poly(lactic-co-glycolic acid) (PLGA) ICC scaffolds, the potential to control the diameter of the cavities and the channels connecting the cavities, the compressive modulus of the scaffolds, and the biocompatibility of scaffolds in vitro.

\section{MATERIALS AND METHODS}

\section{Preparation of colloidal crystals}

One gram of uniformly sized soda lime microspheres (Duke Scientific Corporation, Palo Alto, CA) were added to ethylene glycol (Sigma, St. Louis, MO) in a dropper bottle. Molds were assembled by securing a glass Pasteur pipette to a glass vial (7-mm inner diameter) so that the pipette tip hung centered within the vial. Each mold was filled with ethylene glycol and positioned in an ultra-sonic bath. Two drops of the microsphere solution was dropped into the pipette every $15 \mathrm{~min}$, so the microspheres slowly settled onto the bottom of the vial and assembled into a colloidal crystal (CC) under gentle sonication. After the desired volume of microspheres was added to the mold, the pipette was detached, and the microspheres and vial were left under sonication for an additional hour. The vial was then heated at $160^{\circ} \mathrm{C}$ overnight to evaporate all ethylene glycol before heating for $3 \mathrm{~h}$ to anneal the microspheres together, forming a solid CC. CCs were constructed of 100-, 200-, and 330- $\mu \mathrm{m}$ microspheres to yield ICCs with three different cavity sizes.

The annealing temperatures were adjusted in two separate experiments. In the first experiment, the annealing temperature was varied to explore the controllability of the diameter of interconnecting channels. Here, 100- $\mu \mathrm{m} \mathrm{mi-}$ crospheres were annealed at $660^{\circ} \mathrm{C}, 670^{\circ} \mathrm{C}, 680^{\circ} \mathrm{C}$, and $690^{\circ} \mathrm{C} ; 200-\mu \mathrm{m}$ microspheres were annealed at $670^{\circ} \mathrm{C}$, $680^{\circ} \mathrm{C}, 690^{\circ} \mathrm{C}$, and $700^{\circ} \mathrm{C} ; 330-\mu \mathrm{m}$ microspheres were annealed at $690^{\circ} \mathrm{C}, 700^{\circ} \mathrm{C}$, and $710^{\circ} \mathrm{C}$, and $720^{\circ} \mathrm{C}$. Based on the results from the first experiment, six combinations of microsphere size and annealing temperatures were chosen to produce scaffolds with different-sized cavities and interconnecting channels for cell culture. Microspheres with a $100-\mu \mathrm{m}$ diameter were annealed at $670^{\circ} \mathrm{C}$ and $690^{\circ} \mathrm{C}$, and 200- and 330- $\mu \mathrm{m}$ microspheres were annealed at $690^{\circ} \mathrm{C}$ and $700^{\circ} \mathrm{C}$. These temperatures were chosen based on stability of $\mathrm{CCs}$ and the variety of resulting channel sizes.

\section{Preparation of ICC scaffolds}

CCs were removed from vials and placed into a $0.1-\mathrm{g} / \mathrm{mL}$ solution of 85:15 PLGA (Lactel Absorbable Polymers, Pelham, AL) in methylene chloride. The CCs were centrifuged for $10 \mathrm{~min}$ at $5900 \mathrm{rpm}$ to infiltrate the PLGA solution. The infiltrated CCs were allowed to dry at room temperature overnight and then placed under vacuum for $24 \mathrm{~h}$. The edges of each infiltrated CC were scraped lightly with a razor blade to remove surface PLGA and expose the microspheres. Next, the infiltrated CCs were stirred in a $5 \%$ hydrofluoric acid (HF) solution for 2 days, changing HF solution once, and then stirred in a $1.0 \mathrm{~N}$ hydrochloric acid $(\mathrm{HCl})$ solution for 1 day. The $\mathrm{HF} / \mathrm{HCl}$ cycle was repeated, rinsing with 
distilled water $=$ between cycles. To ensure that all HF was rinsed from the scaffolds, resulting ICCs were washed in phosphate buffered saline (PBS), changing solution until the $\mathrm{pH}$ remained stable at 7.4.

\section{Characterization of ICCs}

The order, overall structure, and diameter of interconnecting channels were characterized for scaffolds of 100-, $200-$, and $330-\mu \mathrm{m}$ cavity diameters. The HCP order of CCs for each microsphere size was observed using scanning electron microscopy (SEM). In the first annealing temperature experiment, ICCs were characterized using SEM to observe overall order and how the interconnecting channel size varied with microsphere annealing temperature. For each microsphere size, two ICCs resulting from each microsphere size and annealing temperature combination were examined, and 10 interconnecting channels were measured on each scaffold. The diameters of interconnecting channels were analyzed using SEM images using the public domain NIH Image program (developed at the U.S. National Institutes of Health and available t http://rsb.info.nih.gov/ nih-image/).

\section{Mechanical properties}

Four ICCs constructed from 100-, 200-, and 330- $\mu \mathrm{m} \mathrm{mi-}$ crospheres with annealing temperatures of $670^{\circ} \mathrm{C}, 690^{\circ} \mathrm{C}$, and $700^{\circ} \mathrm{C}$, respectively, were mechanically tested in compression using a 100Q Universal Test System Mechanical Properties Tester (TestResources Inc, Shakopee, MN). ICCs were tested in a dry state at room temperature. The specimens were compressed at a rate of $0.01 \mathrm{~mm} / \mathrm{s}$, and the compressive modulus was defined as the slope of the linear portion of the stress-strain curve.

\section{Cell culture}

Human fetal osteoblast (hFOB) cell line 1.19 (CRL11372) was purchased from American Tissue Culture Corporation (ATCC, Manassas, VA) and grown as recommended by ATCC in 45\% Ham's F12 medium, 45\% Dulbecco's modified Eagle medium, and 10\% fetal bovine serum (Gibco, Frederick, MD) supplemented with $1 \%$ antibiotic. Cells were grown at $37^{\circ} \mathrm{C}$ and $5 \%$ carbon dioxide $\left(\mathrm{CO}_{2}\right)$, with the medium changed every 2 to 3 days.

\section{Cell loading and culture on scaffolds}

Twelve ICCs, $7 \mathrm{~mm}$ in diameter and $1.2 \mathrm{~mm}$ in height, were constructed for each of the six cavity size and annealing temperature combinations mentioned above. Scaffolds were sterilized and wetted with ethanol for $1 \mathrm{~h}$. Scaffolds were then centrifuged twice in fresh PBS at $900 \mathrm{rpm}$ for $5 \mathrm{~min}$ and then placed in a well of a 12-well plate. hFOB 1.19 cells were stained with trypan blue (Sigma) and counted before being suspended in medium and distributed into wells at $4.6 \times 10^{4}$ cells/scaffold. Cells and scaffolds were incubated at $37^{\circ} \mathrm{C}$ and $5 \% \mathrm{CO}_{2}$. Three scaffolds of each cavity size and annealing temperature were removed at 1, 5, 9, and 12 days for viability staining, and three scaffolds were removed for double-stranded DNA (dsDNA) quantification.

In a second set of cell culture experiments aimed at quantifying and examining the osteoblast phenotype, 20 scaffolds of the following cavity sizes and annealing temperatures were used: $100 \mu \mathrm{m}, 670^{\circ} \mathrm{C} ; 200 \mu \mathrm{m}, 690^{\circ} \mathrm{C}$; $330 \mu \mathrm{m}, 700^{\circ} \mathrm{C}$. Scaffolds were prepared as described above with ethanol and PBS treatment and then placed into wells of a 96-well plate before cells were seeded at densities of $2.0 \times 10^{4}$ cells/scaffold. Scaffolds were inserted to 96 -well plates because the scaffolds would cover the entire bottom area of the wells, increasing the consistency of cell seeding into the scaffolds rather than onto the bottom of the well plate. Cells were incubated at $37^{\circ} \mathrm{C}$ and $5 \% \mathrm{CO}_{2}$. After 1 day, scaffolds were transferred to a 24 -well plate to increase the amount of medium surrounding cells. Two scaffolds were removed after $3 \mathrm{~h}$, and six scaffolds were removed on days 1,4 , and 7 . At all time points, two scaffolds were used for F-actin and collagen type I staining, and during the later three time points, four scaffolds were used for dsDNA and alkaline phosphatase (ALP) activity.

\section{Staining and confocal microscopy for viability, F-actin, and collagen type I}

Cells on each of the three scaffolds removed at each time point for viability assessment were stained with $2 \mu \mathrm{M}$ of calcein $\mathrm{AM}$ and $4 \mu \mathrm{M}$ of ethidium homodimer-1 using a Live/Dead Viability/Cytotoxicity Kit (Invitrogen Corporation, Carlsbad, CA).

Scaffolds for F-actin and collagen type I staining were removed at each time point, fixed with $4 \%$ formaldehyde for $10 \mathrm{~min}$ at room temperature, rinsed in PBS twice, permeablized with $0.1 \%$ Triton $\mathrm{X}-100$ for 3 to $5 \mathrm{~min}$, rinsed twice more with PBS, and then immediately treated for visualization of collagen or F-actin. Cells in scaffolds used for Factin staining were incubated for $30 \mathrm{~min}$ with rhodamine phalloidin (Invitrogen Corporation). Scaffolds used for antibody immunohistological staining of collagen were stored in a $40-\mu \mathrm{g} / \mathrm{mL}$ solution of biotin-labeled polyclonal goat anti-collagen type I (Millipore, Billerica, MA) at $4{ }^{\circ} \mathrm{C}$ overnight. Samples were rinsed twice in PBS and incubated in a $10-\mu \mathrm{g} / \mathrm{mL}$ solution of fluorescein isothiocyanatestreptavidin (MP Biomedicals, Solon, OH) for $1 \mathrm{~h}$ at $37^{\circ} \mathrm{C}$. All samples were viewed immediately or stored at $4^{\circ} \mathrm{C}$ protected from light and were examined using a Leica TCS SP2 confocal laser scanning microscope with $10 \times$ and $20 \times$ objectives.

\section{Scanning electron microscopy}

Samples for SEM were fixed in $2 \%$-volume glutaraldehyde solution overnight and then washed in sodium 
FIG. 1. Scanning electron microscopy images of colloidal crystals made from microspheres of diameter (A) $100 \mu \mathrm{m}$, (B) $200 \mu \mathrm{m}$, and (C) $330 \mu \mathrm{m}$. Scale bar $=200 \mu \mathrm{m}$.

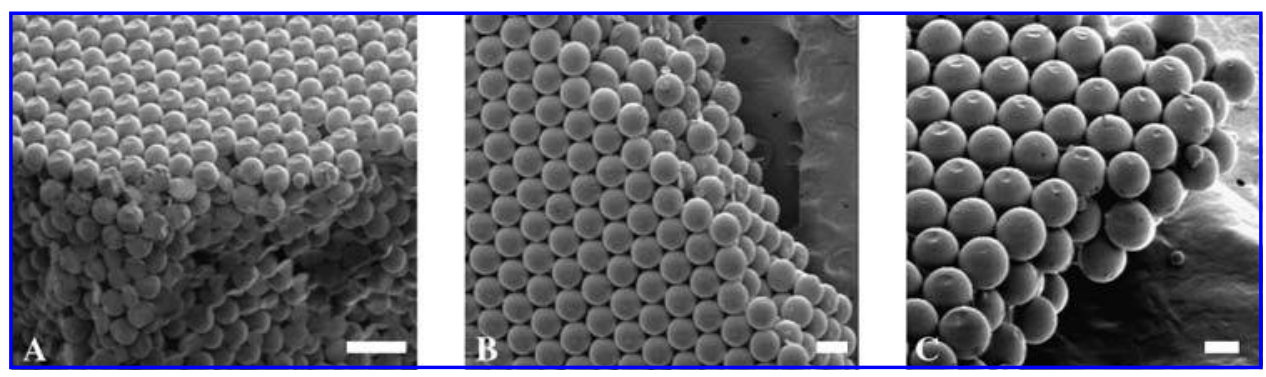

cacodylate solution for $1 \mathrm{~h}$. Samples were dehydrated through an ethanol series with concentrations of $20 \%, 50 \%$, $70 \%, 90 \%$, and $100 \%$, rinsing in each for $20 \mathrm{~min}$. They were then freeze-dried and gold-coated. SEM observations were performed using a Nova Nanolab Dualbeam Focussed Ion Beam Workstation (FEI Company, Hillsboro, OR) and SEM at $15 \mathrm{kV}$.

\section{dsDNA quantification}

Scaffolds slated for dsDNA quantification were washed with PBS, sonicated in $500 \mu \mathrm{L}$ of passive lysis buffer (Promega Corporation, Madison, WI), and stored at $-70^{\circ} \mathrm{C}$ until quantification, when samples were thawed and centrifuged for $10 \mathrm{~min}$ at $10,000 \mathrm{rpm}$. Of the supernatant, $50 \mu \mathrm{L}$ was prepared for dsDNA quantification using Quant-iT PicoGreen dsDNA Reagent (Invitrogen Corporation), according to the manufacturer's protocol. Sample fluorescence was measured at $520 \mathrm{~nm}$ using excitation at $480 \mathrm{~nm}$ on a Synergy 2 Multi-Detection Microplate Reader (Biotek, Winooski, VT).

\section{ALP activity}

ALP activity was assessed using a colorimetric endpoint assay measuring the conversion of colorless 4-nitrophenyl phosphate (p-NPP) (Sigma) to 4-nitrophenol (p-NP) (Sigma) using ALP. ${ }^{22}$ Samples were lysed and centrifuged as described above. Of the supernatant, $50 \mu \mathrm{L}$ was used to quantify dsDNA, and $10 \mu \mathrm{L}$ was incubated with $40 \mu \mathrm{L}$ of $\mathrm{p}-\mathrm{NPP}$ $(20 \mathrm{mM})$ for $30 \mathrm{~min}$ at $37^{\circ} \mathrm{C}$. The reaction was stopped by adding $50 \mu \mathrm{L}$ of $0.1 \mathrm{~N}$ sodium hydroxide, and sample absorbance was determined at $405 \mathrm{~nm}$ on a microplate reader. The activity of enzyme present was quantified by comparison with a standard curve. ALP activity was normalized to cell number using dsDNA assay results.

\section{Statistical analysis}

In all graphs, data are represented as the average of the indicated sample sizes \pm standard deviations. A Student $t$-test was performed to determine statistical significance $(p<0.05)$.

\section{RESULTS}

\section{Characterization of ICC scaffolds}

The chosen range of annealing temperatures was selected to provide a $\mathrm{CC}$ that was stable enough to be handled and provide interconnectivity between adjacent microspheres. The upper limit of the temperature range was chosen so that microspheres were not completely melted, which would inhibit the infiltration of PLGA in spaces between microspheres.

Highly ordered CCs were assembled from uniformly sized soda lime microspheres 100,200 , and $330 \mu \mathrm{m}$ in diameter (Fig. 1). For all three sizes, microspheres were arranged in an HCP array, as observed in the structure cross-sections. After infiltration with PLGA, beads were completely removed using $\mathrm{HF} / \mathrm{HCl}$, resulting in ICC structures with a degree of
FIG. 2. Scanning electron microscopy images of poly(lactic-coglycolic acid)inverted colloidal crystals resulting from colloidal crystals of microspheres of diameter (A) $100 \mu \mathrm{m}$, (B) $200 \mu \mathrm{m}$, and (C) $330 \mu \mathrm{m}$. Scale bar $=200 \mu \mathrm{m}$.

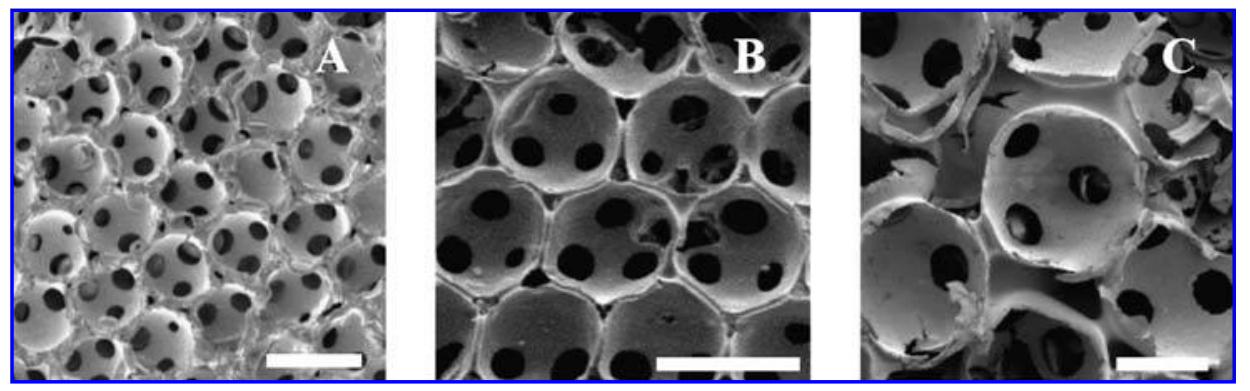




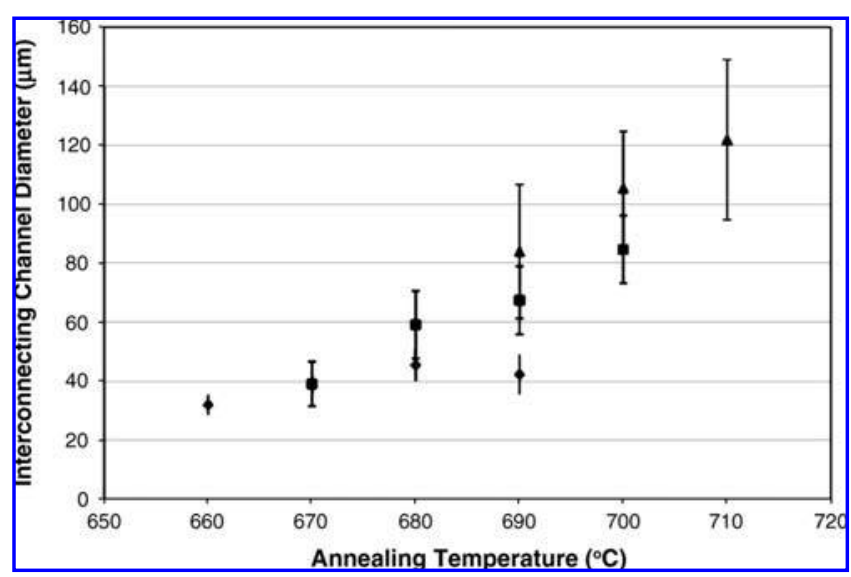

FIG. 3. Interconnecting channel diameters for inverted colloidal crystals with 100-, 200-, and 330- $\mu \mathrm{m}$ cavities with varying microsphere annealing temperatures.

order similar to that of the CC templates (Fig. 2). The cavities were uniform in size and showed interconnectivity with all adjacent cavities.

\section{Channel size control using annealing temperature}

To explore the degree of control over the diameter of interconnecting channels using CC annealing temperature, each microsphere size was annealed at four temperatures. Several $100-\mu \mathrm{m}$ CCs annealed at temperatures higher than $690^{\circ} \mathrm{C}$ were found to anneal to the vial that served as the $\mathrm{CC}$ mold and could not be removed for PLGA infiltration. It was assumed that annealing at temperatures higher than $690^{\circ} \mathrm{C}$ caused excessive melting of $100-\mu \mathrm{m}$ microspheres. The edges of 200- $\mu \mathrm{m}$ CCs annealed at temperatures lower than $670^{\circ} \mathrm{C}$ crumbled when removed from the vial mold; it is likely that lower temperatures did not yield sufficient annealing to provide a secure structure for handling. For 330$\mu \mathrm{m}$ microspheres, annealing temperatures of $670^{\circ} \mathrm{C}$ and $680^{\circ} \mathrm{C}$ were attempted, but the resulting CCs were not stable enough to be handled without breaking; thus it was decided that higher temperatures would be explored. Additionally, because $330-\mu \mathrm{m} \mathrm{CCs}$ annealed at $720^{\circ} \mathrm{C}$ were melted and cracked, ICCs were not constructed at this temperature. ICCs from CCs of each microsphere diameter and annealing temperature were characterized using SEM. The interstitial channel diameters were measured to ensure sufficient size to allow cell migration throughout the scaffold. Figure 3 shows

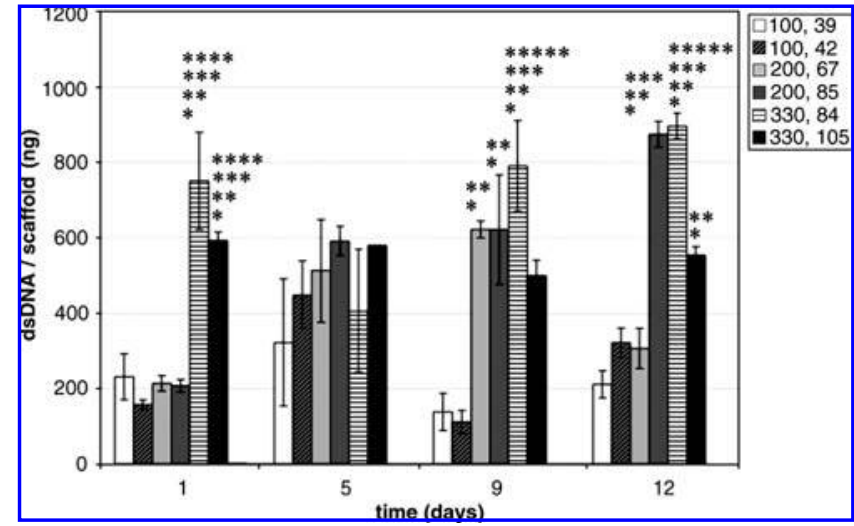

FIG. 4. Double strand DNA present in scaffolds $(n=3)$ of different cavity and interconnecting space diameters for culture up to 12 days. The legend indicates the following: cavity diameter $(\mu \mathrm{m})$, interconnecting space diameter $(\mu \mathrm{m})$. (*Statistically significant $(p<0.05)$ compared with 100, 39 for the indicated culture period; $* *$ Statistically significant $(p<0.05)$ compared with 100,42 for the indicated culture period; $* * *$ Statistically significant $(p<0.05)$ compared with 200,67 for the indicated culture period; $* * * *$ Statistically significant $(p<0.05)$ compared with 200,85 for the indicated culture period; $* * * * *$ Statistically significant $(p<0.05)$ compared with 330,105 for the indicated culture period.)

the average diameters for ICCs resulting from each microsphere size and annealing temperature.

\section{Mechanical properties}

The compressive moduli of PLGA ICCs resulting from each microsphere size are displayed in Table 1 . The compressive moduli of ICCs with $100-$ and $200-\mu \mathrm{m}$ cavity diameters are slightly higher than those with $330-\mu \mathrm{m}$ cavities.

\section{dsDNA quantification}

Two annealing temperatures were chosen for each cavity size to determine the effects of cavity and channel diameter on osteoblast cell culture. dsDNA quantification for each of these six scaffold cavity and annealing temperature sizes is displayed in Figure 4 for up to 12 days. There was a trend toward more cells with larger cavity diameters, and proliferation did not seem significant over the culture period.

Table 1. Compressive Modulus of Poly(Lactic-Co-Glycolic Acid) Inverted Colloidal Crystals Constructed Using 100-, 200-, AND 330-Mm Microspheres at the Indicated Annealing Temperatures $(N=4)$

\begin{tabular}{lccc}
\hline & $100 \mu \mathrm{m}, 670^{\circ} \mathrm{C}$ & $200 \mu \mathrm{m}, 690^{\circ} \mathrm{C}$ & $330 \mu \mathrm{m}, 700^{\circ} \mathrm{C}$ \\
Compressive Modulus ( MPa) & $55.24 \pm 17.57$ & $63.63 \pm 14.47$ & $54.80 \pm 30.98$ \\
\hline
\end{tabular}


FIG. 5. Confocal microscopy images of human fetal osteoblast cell line 1.19 culture on inverted colloidal crystals with cavity sizes and annealing temperatures of (A) $100 \mu \mathrm{m}, 670^{\circ} \mathrm{C}$; (B) $200 \mu \mathrm{m}, 700^{\circ} \mathrm{C}$; (C) $330 \mu \mathrm{m}$, $700^{\circ} \mathrm{C}$; (D) $100 \mu \mathrm{m}, 690^{\circ} \mathrm{C}$; (E) $200 \mu \mathrm{m}, 700^{\circ} \mathrm{C}$; and (F) $330 \mu \mathrm{m}$, $700^{\circ} \mathbf{C}$. A-C are images of cultures after 1 day, and $\mathbf{D}-\mathbf{F}$ are images taken after 12 days of culture. Scale bar $=200 \mu \mathrm{m}$. Color images available online at www.liebertonline.com/ten.

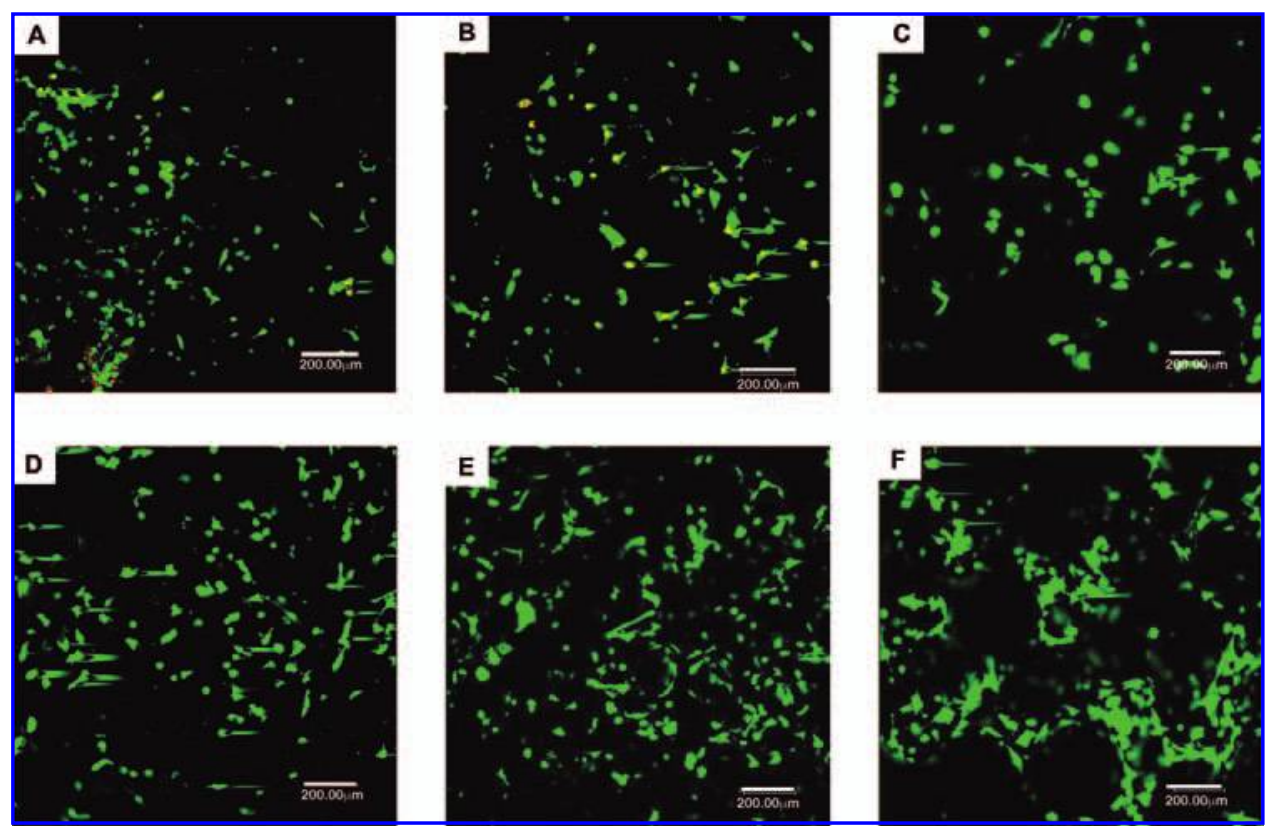

\section{Biocompatibility of PLGA ICC scaffolds}

Confocal microscopy of hFOB 1.19 cultures on scaffolds $1,5,9$, and 12 days after seeding onto the scaffolds revealed attachment, viability, and proliferation of human osteoblasts for up to 12 days on the scaffolds, as displayed in Figure 5. Most cells remained viable for the duration of the culture period. SEM images (Fig. 6) reveal variety in cell morphologies, ranging from fully spread and attached cells to rounded cells with less area of attachment. Confocal microscopy images of F-actin staining using rhodamine phalloidin for cell cultures of $3 \mathrm{~h}$ and 1 and 4 days are shown in Figure 7. F-actin staining reveals that, at $3 \mathrm{~h}$, cells are rounded, probably because they have not attached to the scaffold, and most F-actin is localized near the cell mem- brane. After 1 day, cells have attached to the scaffold and show more uniform F-actin distribution, because their cytoskeletal structure is spread, indicating interaction and contact with the scaffolds.

\section{Maintenance of osteoblast phenotype}

Anti-collagen antibody staining is shown in Figure 8. Collagen type I antibody staining did not reveal any collagen in the structure at day 1 , but by day 4 , the presence of collagen was observed within or outside of the cells, indicating an osteoblast phenotype. ALP activity for each scaffold cavity size at 1, 4, and 7 days is shown in Figure 9. ALP activity, however, seems slightly higher for larger cavity sizes, and an upregulation was observed over time.
FIG. 6. Scanning electron microscopy images of human fetal osteoblast cell line 1.19 on inverted colloidal crystals demonstrating variety of cell morphologies. Cavity diameters and annealing temperatures for pictured scaffolds are (A) $200 \mu \mathrm{m}, 690^{\circ} \mathrm{C}$ and (B) $330 \mu \mathrm{m}$, $690^{\circ} \mathrm{C}$. Both images are after 5 days of culture.

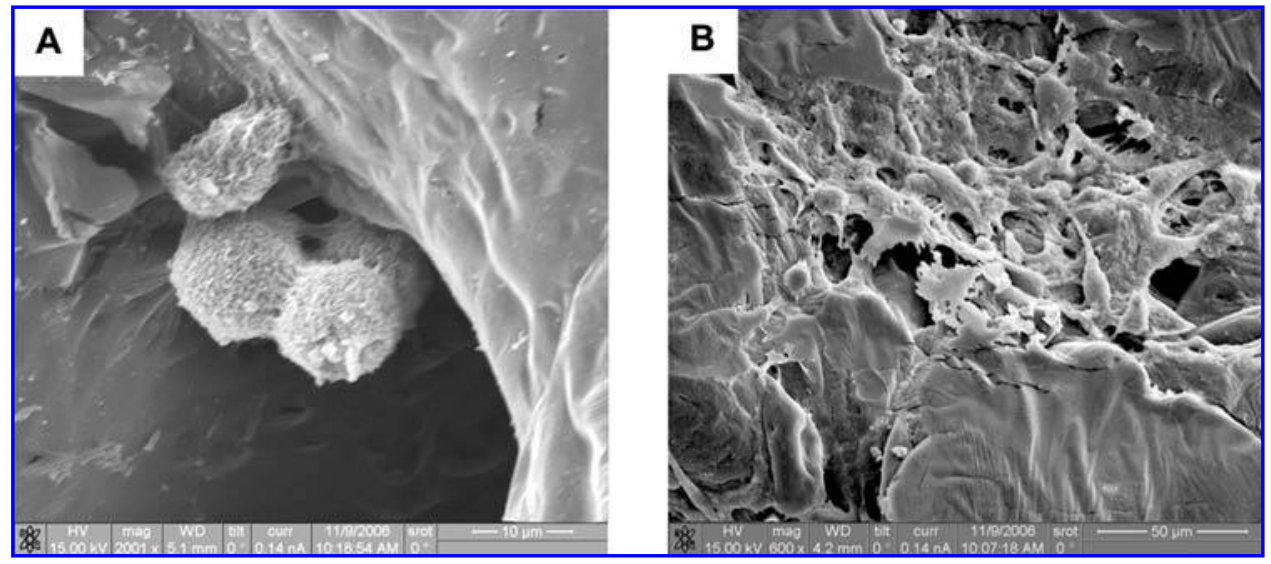




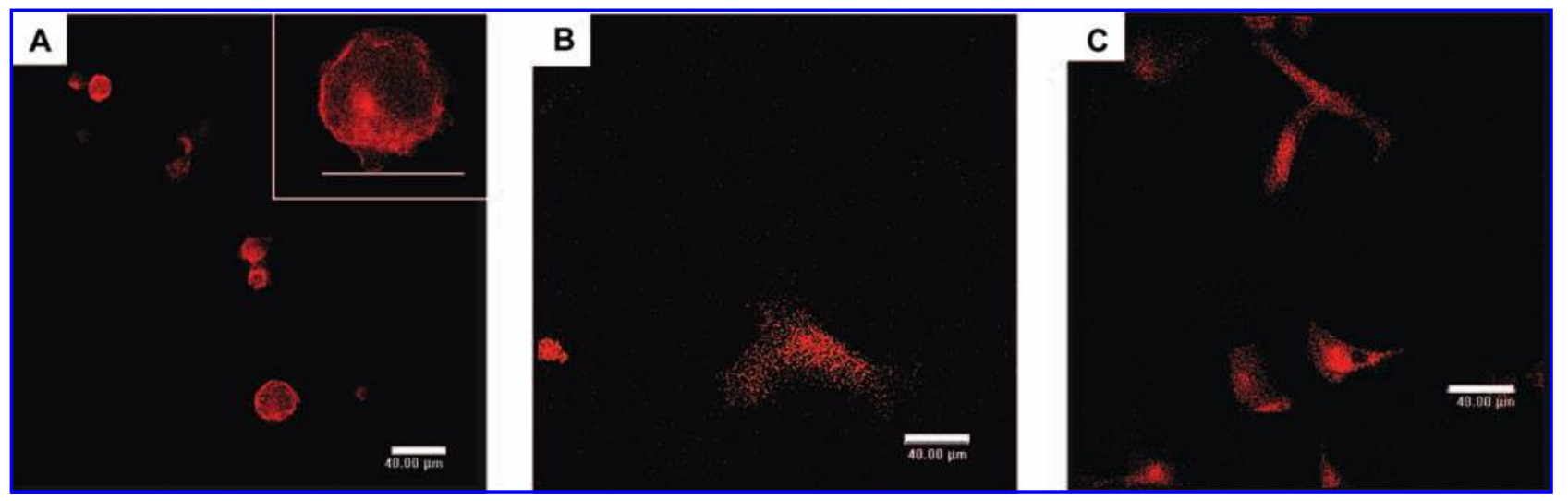

FIG. 7. Confocal microscopy images of human fetal osteoblast cell line 1.19 on inverted colloidal crystals stained for actin after (A) $3 \mathrm{~h}$ (inset, greater magnification of bottommost cell in image), (B) 1 day, and (C) 4 days. Color images available online at www .liebertonline.com/ten.

\section{DISCUSSION}

The internal architecture of a scaffold defines the fundamental characteristics of the construct. For example, the geometry of a scaffold define the surface area for cell migration and proliferation, void volume for tissue and vascular invasion in vivo, and bulk scaffold (i.e., mechanical) properties. ${ }^{23-26}$ Control over scaffold internal architecture is important to meet specific design criteria. ${ }^{27,28}$ Principally, we aimed to prove control over scaffold cavity size and the size of the interconnecting channels between cavities, which directly relate to surface area, void volume, and mechanical properties.

Microsphere size, HCP arrangement, and CC annealing temperature primarily control the cavity and interconnecting channel sizes of ICCs. Unlike hydrogel, PLGA does not significantly expand into cavities when microspheres are removed; therefore, the microsphere diameter accurately defines the resulting cavity diameter. The combination of $\mathrm{HCP}$ structure and microsphere annealing provides full interconnectivity throughout the ICC scaffold. The highly ordered structure of CCs guarantees connectivity of each cavity to 12 neighboring cavities. Annealing not only gives stability to the CC structure, but also provides the sites of connection between cavities in ICCs. Annealing temperature regulation is essential to ensure complete PLGA infiltration and scaffold interconnectivity and to regulate the interconnecting channel size.

Pore size is of interest in controlling tissue growth, particularly bone, into scaffolds. ${ }^{29,30}$ In vivo, it is generally believed that bone ingrowth and vascularization are maximized over fibrous or connective tissue ingrowth in scaffolds in pore diameters greater than $100 \mu \mathrm{m} ;{ }^{31,32}$ however, this has been debated, with research indicating that smaller pore diameters encourage bone growth. ${ }^{33}$ Others have shown that bone growth occurs via chondrogenesis in $100-\mu \mathrm{m}$ pores

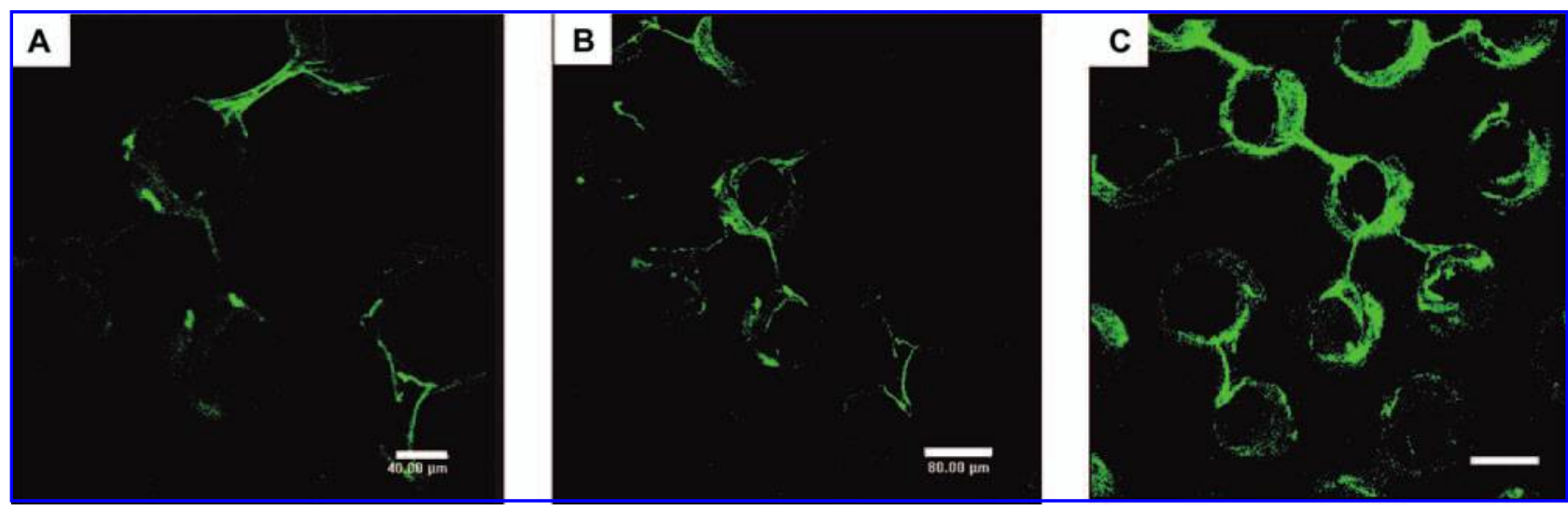

FIG. 8. Confocal microscopy images of human fetal osteoblast cell line 1.19 on inverted colloidal crystals stained for collagen type I after 4 days of culture. (A) Scale bar $=40 \mu \mathrm{m}$. (B) and (C) Scale bar $=80 \mu \mathrm{m}$. Color images available online at www.liebertonline.com/ten. 


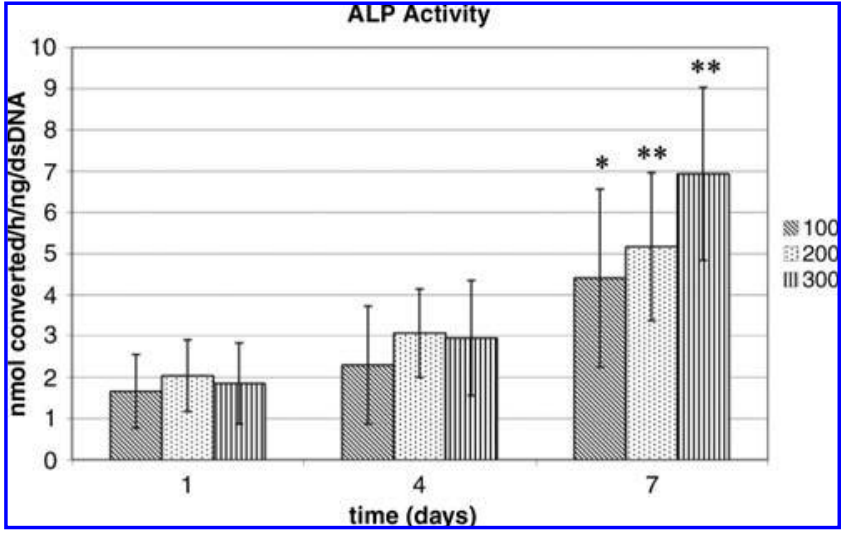

FIG. 9. Alkaline phosphatase activity measured in scaffolds $(n=4)$, normalized according to double-strand DNA levels in scaffolds. (*Statistically significant $(p<0.05)$ compared with day 1. **Statistically significant $(p<0.05)$ compared with days 1 and 4 for the indicated cavity diameter.)

within hydroxyapatite scaffolds, yet with $350-\mu \mathrm{m}$ pores, osteogenesis occurs directly. ${ }^{34-36}$ In vitro, it has been shown that larger pore sizes correlate with earlier osteoblast differentiation. ${ }^{26}$ There is a need for a systematic method of varying pore diameters to determine optimal bone growth conditions.

In this study, diameters of 100,200 , and $330 \mu \mathrm{m}$ were chosen to demonstrate the ability to construct PLGA ICCs with a range of cavity diameters that would support nutrient and waste diffusion and convection in vitro and in vivo, as well as tissue and vascular in-growth in vivo. Additionally, architectures in this range have been shown to promote osteoblast or bone growth. ${ }^{37,38}$

For each cavity size, the interconnecting channels were large enough to facilitate cell migration throughout the scaffold. Although there is some overlap in the size of interconnecting channels at different annealing temperatures, there is an obvious trend of higher annealing temperature causing larger interconnecting channel diameters. This is important for this type of scaffold because this experiment demonstrates that the diameter of the cavities and the diameter of the channels connecting them can be controlled during the preparation of the scaffold. More melting of microspheres at higher temperatures, which provides a greater void space between cavities, causes the larger channel diameters. A slightly greater range of channel diameters exists for each temperature with $330-\mu \mathrm{m}$ microspheres. This is most likely due to the greater size distribution of $330-\mu \mathrm{m}$ microspheres (4.9\%) than with $100-\mu \mathrm{m}(3.1 \%)$ and $200-\mu \mathrm{m}$ (2.9\%) microspheres, which will cause less-ordered packing and a less consistent degree of contact between microspheres in CCs. Additionally, the larger microsphere size causes faster settling of microspheres during CC construction, which may hinder ordered packing, despite sonication. In each case, the range of interconnecting channel diameters may also be attributed to uneven heat distribution throughout the CC. Adjusting the time of annealing may increase the control over degree of annealing to decrease the variation in interconnecting channel diameters. Despite the range of interconnecting channel diameters, the general linear increase in channel diameter with higher annealing temperature demonstrates an additional level of control over ICC scaffold properties that has not been demonstrated in many other scaffold fabrication techniques.

The targeted functional outcome of bone tissue engineering is to produce a scaffold-cell construct that has mechanical properties supporting the loads of daily life. ${ }^{39}$ Overall, the ICC geometry possesses an advantage over traditional porous scaffolds because of its complete interconnectivity at lower porosities. The compressive moduli are greater than those of other porous PLGA scaffolds fabricated using a stochastic arrangement of porogens, ${ }^{40,41} \mathrm{In}$ the compressive moduli of the scaffolds are in the range of other polymer scaffolds fabricated using SFF, which can be designed to meet specific mechanical properties. ${ }^{23,42}$ Considering that the majority of current scaffolds are now composed of composite materials to improve mechanical properties, the ICC scaffolds have great potential to approach the mechanical properties of bone. Better mechanical properties may be obtained using additional infiltration steps or infiltrating with a stronger biodegradable composite material. An additional infiltration step for the $330-\mu \mathrm{m}$ or larger CCs may be especially advantageous in obtaining morphological similarity to trabecular bone, which has trabeculae of 80 to $280 \mu \mathrm{m}$ in thickness and more than $450 \mu \mathrm{m}$ of separation between trabeculae. ${ }^{43}$

Confocal microscopy images reveal that cells were able to attach and inhabit the ICC scaffolds for up to 12 days, and the abundance of calcein AM-stained cells (green) indicate that viability was strong. Cell survival over several weeks is especially important to establish because of the harsh acid treatment required to remove the microspheres. Figure 5E and $\mathrm{F}$ shows how cells have begun to coat the cavity walls and appear to have infiltrated beyond the outer layer of cavities.

The variety of cell morphologies displayed in Figure 6 indicates that, although cells adhere to the PGLA ICC scaffold, cell adhesion could be improved to achieve more consistently spread morphologies. Greater cell attachment will also encourage cell migration within the scaffold, yielding a more uniform population throughout the structure. F-actin staining revealed that the rounded morphologies with actin localized to the cell membrane were demonstrated at times close to seeding and that flattened morphologies with a uniform cytoskeleton were observed after 1 day. The rounded morphologies observed in SEM images may be cells that have recently attached, migrated, or replicated. Still, collagen was not observed $3 \mathrm{~h}$ or 1 day after seeding, indicating that surface modification that enhances or speeds cell spreading may be beneficial in increasing bone production within the scaffold. 
dsDNA quantification revealed a trend that more cells could be found on scaffolds of larger pore sizes, probably because, as the pore sizes and interconnecting channel sizes increase, the cavity openings on the surface of the scaffold are larger, leading to greater cell seeding efficiency. Additionally, there was a trend toward greater ALP activity with greater cavity sizes, as well as greater ALP activity over time. ALP activity was normalized to dsDNA, indicating that cells were more active over time and that the increased activity was not due to greater numbers of cells present. Because more cells could be seeded into larger pores, perhaps their proximity within spherical pores signal osteogenesis. Additionally, it is likely that the larger pore sizes allow for greater circulation of nutrients, signaling factors, and metabolites.

Because the quantity of dsDNA did not increase greatly over time for most cavity and interconnecting channel sizes (Fig. 4), it is likely that cells were in a differentiated state, rather than a proliferative state. Low proliferation, increasing ALP activity over time, and the presence of collagen type I are important parameters to consider, particularly when using the hFOB 1.19 pre-osteoblast cell line, because these parameters are indicators of osteoblast differentiation and mineralization. ${ }^{22,44,45}$ Because this cell line has a temperature-dependent phenotype, meaning that, at $33.5^{\circ} \mathrm{C}$, cells rapidly replicate and that, at $39.5^{\circ} \mathrm{C}$, cells display little or no cell division. ${ }^{46}$ In these studies, it was shown that, at the incubation temperature used, $37^{\circ} \mathrm{C}$, there was low cell replication, as well as factors indicative of bone production.

These studies support conclusions that cavity sizes larger than $100 \mu \mathrm{m}$ are ideal for bone growth, but further study must be performed. Still, one must also remember that with larger pore sizes, mechanical properties are likely to decrease. In vivo studies may be more indicative of effects of scaffold feature sizes, because larger quantities of cells, tissues, and circulating fluids are in contact with the scaffold than during in vitro studies.

\section{CONCLUSIONS}

A new geometry of biodegradable ICC scaffolds was demonstrated, with consistent geometries and cavity sizes of 100,200 , and $330 \mu \mathrm{m}$. These studies indicated trends that larger pore sizes may increase cell growth and bone production. PLGA ICC scaffolds have great potential for bone tissue engineering applications because they possess a regular structure for good mechanical properties and uniform cell loading and retention. The spherical cavities also provide maximal surface area and variable diameters to support tissue and vascular invasion.

The ICC structure has broad applications in bone tissue engineering in that it can be incorporated into molds with more complex geometries designed to fit anatomical shapes, for robust mechanical properties, or with architectures ideal for tissue invasion. For example, the ICC structure can be incorporated into molds designed with complex internal architecture for better mechanical functionality and made using SFF fabrication methods. This incorporation can take advantage of the mechanical and geometric functionality of the SFF scaffold while increasing its surface area and topography. For this application, CC construction with smaller microspheres might be desired to facilitate greater order when depositing microspheres into molds with their own internal architecture while providing larger pores in between struts with ICC internal geometries.

\section{ACKNOWLEDGMENTS}

This work was supported by the National Institutes of Health (NIH) grant 5R01EB007350-02. The authors would like to thank Dr. Michael Solomon and the Solomon group (University of Michigan, Ann Arbor, MI) for their assistance in using the confocal scanning laser microscope.

\section{REFERENCES}

1. Langer, R., and Vacanti, J.P. Tissue engineering. Science. 260, 920, 1993.

2. Laurencin, C.T., Ambrosio, A.M., Borden, M.D., and Cooper, J.A., Jr. Tissue engineering: orthopedic applications. Annu Rev Biomed Eng. 1, 19, 1999.

3. Liu, X., and Ma, P.X. Polymeric scaffolds for bone tissue engineering. Ann Biomed Eng. 32, 477, 2004.

4. Taboas, J.M., Maddox, R.D., Krebsbach, P.H., and Hollister, S.J. Indirect solid free form fabrication of local and global porous, biomimetic and composite 3D polymer-ceramic scaffolds. Biomaterials. 24, 181, 2003.

5. Lin, C.Y., Schek, R.M., Mistry, A.S., Shi, X., Mikos, A.G., Krebsbach, P.H., and Hollister, S.J. Functional bone engineering using ex vivo gene therapy and topology-optimized, biodegradable polymer composite scaffolds. Tissue Eng. 11, 1589, 2005.

6. Temenoff, J.S., and Mikos, A.G. Formation of highly porous biodegradable scaffolds for tissue engineering. Electron J Biotechnol. 3, 114, 2000.

7. Dunn, J.C., Chan, W.Y., Cristini, V., Kim, J.S., Lowengrub, J., Singh, S., and Wu, B.M. Analysis of cell growth in threedimensional scaffolds. Tissue Eng. 12, 705, 2006.

8. Jones, J.R., Ehrenfried, L.M., and Hench, L.L. Optimising bioactive glass scaffolds for bone tissue engineering. Biomaterials. 27, 964, 2006.

9. Li, W.J., Tuli, R., Okafor, C., Derfoul, A., Danielson, K.G., Hall, D.J., and Tuan, R.S. A three-dimensional nanofibrous scaffold for cartilage tissue engineering using human mesenchymal stem cells. Biomaterials. 26, 599, 2005.

10. Nam, Y.S., and Park, T.G. Biodegradable polymeric microcellular foams by modified thermally induced phase separation method. Biomaterials. 20, 1783, 1999.

11. Hollister, S.J. Porous scaffold design for tissue engineering. Nat Mater. 4, 518, 2005.

12. Rezwan, K., Chen, Q.Z., Blaker, J.J., and Boccaccini, A.R. Biodegradable and bioactive porous polymer/inorganic 
composite scaffolds for bone tissue engineering. Biomaterials. 27, 3413, 2006.

13. Yang, S., Leong, K.F., Du, Z., and Chua, C.K. The design of scaffolds for use in tissue engineering. Part II. Rapid prototyping techniques. Tissue Eng. 8, 1, 2002.

14. Lee, J.W., Cuddihy, M.J., and Kotov, N.A. 3D cell culture matrices: state of the art. Tissue Eng Part B. 14, 61, 2008.

15. Kotov, N.A., Liu, Y., Wang, S., Cumming, C., Eghtedari, M., Vargas, G., Motamedi, M., Nichols, J., and Cortiella, J. Inverted colloidal crystals as three-dimensional cell scaffolds. Langmuir. 20, 7887, 2004.

16. Lee, J.W., Shanbhag, S., and Kotov, N.A. Inverted colloidal crystals as three-dimensional microenvironments for cellular co-cultures. J Mater Chem. 16, 3558, 2006.

17. Liu, Y., Wang, S., Lee, J.W., and Kotov, N.A. A floating selfassembly route to colloidal crystal templates for 3D cell scaffolds. Chem Mater. 17, 4918, 2005.

18. Shanbhag, S., Woo Lee, J., and Kotov, N. Diffusion in threedimensionally ordered scaffolds with inverted colloidal crystal geometry. Biomaterials. 26, 5581, 2005.

19. Zhang, Y., Wang, S., Eghtedari, M., and Kotov, N.A. Inverted colloidal crystal hydrogel matrices as three-dimensional cellscaffolds. Adv Funct Mater. 15, 725, 2005.

20. Stachowiak, A.N., Bershteyn, A., Tzatzalos, E., and Irvine, D.J. Bioactive hydrogels with an ordered cellular structure combine interconnected macroporosity and robust mechanical properties. Adv Mater. 17, 399, 2005.

21. Jan, E., and Kotov, N.A. Successful differentiation of mouse neural stem cells on layer-by-layer assembled single-walled carbon nanotube composite. Nano Lett. 7, 1123, 2007.

22. Manolagas, S., Burton, D., and Deftos, L. 1,25dihydroxyvitamin $\mathrm{d} 3$ stimulates the alkaline phosphatase activity of osteoblast-like cells. J Biol Chem. 256, 7115, 1981.

23. Williams, J.M., Adewunmi, A., Schek, R.M., Flanagan, C.L., Krebsbach, P.H., Feinberg, S.E., Hollister, S.J., and Das, S. Bone tissue engineering using polycaprolactone scaffolds fabricated via selective laser sintering. Biomaterials. 26, 4817, 2005.

24. Mathieu, L.M., Mueller, T.L., Bourban, P.E., Pioletti, D.P., Muller, R., and Manson, J.A. Architecture and properties of anisotropic polymer composite scaffolds for bone tissue engineering. Biomaterials. 27, 905, 2006.

25. Gomes, M.E., Holtorf, H.L., Reis, R.L., and Mikos, A.G. Influence of the porosity of starch-based fiber mesh scaffolds on the proliferation and osteogenic differentiation of bone marrow stromal cells cultured in a flow perfusion bioreactor. Tissue Eng. 12, 801, 2006.

26. Holtorf, H.L., Datta, N., Jansen, J.A., and Mikos, A.G. Scaffold mesh size affects the osteoblastic differentiation of seeded marrow stromal cells cultured in a flow perfusion bioreactor. J Biomed Mater Res A. 74, 171, 2005.

27. Chen, V.J., and Ma, P.X. The effect of surface area on the degradation rate of nano-fibrous poly(l-lactic acid) foams. Biomaterials. 27, 3708, 2006.

28. Cao, Y., Mitchell, G., Messina, A., Price, L., Thompson, E., Penington, A., Morrison, W., O'Connor, A., Stevens, G., and Cooper-White, J. The influence of architecture on degradation and tissue ingrowth into three-dimensional poly (lactic-co-glycolic acid) scaffolds in vitro and in vivo. Biomaterials. 27, 2854, 2006.

29. Zeltinger, J., Sherwood, J.K., Graham, D.A., Mueller, R., and Griffith, L.G. Effect of pore size and void fraction on cellular adhesion, proliferation, and matrix deposition. Tissue Eng. 7, $557,2001$.

30. Karageorgiou, V., and Kaplan, D. Porosity of 3D biomaterial scaffolds and osteogenesis. Biomaterials. 26, 5474, 2005.

31. Hulbert, S.F., Young, F.A., Mathews, R.S., Klawitter, J.J., Talbert, C.D., and Stelling, F.H. Potential of ceramic materials as permanently implantable skeletal prostheses. J Biomed Mater Res. 4, 433, 1970.

32. Klenke, F.M., Liu, Y., Yuan, H., Hunziker, E.B., Siebenrock, K.A., and Hofstetter, W. Impact of pore size on the vascularization and osseointegration of ceramic bone substitutes in vivo. J Biomed Mater Res A. 2007 Sep 26 [Epub ahead of print].

33. Itala, A.I., Ylanen, H.O., Ekholm, C., Karlsson, K.H., and Aro, H.T. Pore diameter of more than 100 microm is not requisite for bone ingrowth in rabbits. J Biomed Mater Res. 58, 679, 2001.

34. Kuboki, Y., Jin, Q., Kikuchi, M., Mamood, J., and Takita, H. Geometry of artificial ECM: sizes of pores controlling phenotype expression in bmp-induced osteogenesis and chondrogenesis. Connect Tissue Res. 43, 529, 2002.

35. Kuboki, Y., Jin, Q., and Takita, H. Geometry of carriers controlling phenotypic expression in bmp-induced osteogenesis and chondrogenesis. J Bone Joint Surg Am. 83-A Suppl 1, S105, 2001.

36. Flautre, B., Descamps, M., Delecourt, C., Blary, M.C., and Hardouin, P. Porous HA ceramic for bone replacement: role of the pores and interconnections-experimental study in the rabbit. J Mater Sci Mater Med. 12, 679, 2001.

37. Ruhe, P.Q., Hedberg-Dirk, E.L., Padron, N.T., Spauwen, P.H., Jansen, J.A., and Mikos, A.G. Porous poly(dl-lacticco-glycolic acid)/calcium phosphate cement composite for reconstruction of bone defects. Tissue Eng. 12, 789, 2006.

38. Zinger, O., Zhao, G., Schwartz, Z., Simpson, J., Wieland, M., Landolt, D., and Boyan, B. Differential regulation of osteoblasts by substrate microstructural features. Biomaterials. 26, 1837, 2005.

39. Goldstein, S.A. Tissue engineering: functional assessment and clinical outcome. Ann N Y Acad Sci. 961, 183, 2002.

40. Ma, P.X., and Choi, J.W. Biodegradable polymer scaffolds with well-defined interconnected spherical pore network. Tissue Eng. 7, 23, 2001.

41. Shea, L.D., Wang, D., Franceschi, R.T., and Mooney, D.J. Engineered bone development from a pre-osteoblast cell line on three-dimensional scaffolds. Tissue Eng. 6, 605, 2000.

42. Mondrinos, M.J., Dembzynski, R., Lu, L., Byrapogu, V.K., Wootton, D.M., Lelkes, P.I., and Zhou, J. Porogen-based solid freeform fabrication of polycaprolactone-calcium phosphate scaffolds for tissue engineering. Biomaterials. 27, 4399, 2006.

43. Hildebrand, T., Laib, A., Muller, R., Dequeker, J., and Ruegsegger, P. Direct three-dimensional morphometric analysis of human cancellous bone: microstructural data from 
spine, femur, iliac crest, and calcaneus. J Bone Miner Res. 14, 1167, 1999.

44. Kang, M.I., Lee, W.Y., Oh, K.W., Han, J.H., Song, K.H., Cha, B.Y., Lee, K.W., Son, H.Y., Kang, S.K., and Kim, C.C. The short-term changes of bone mineral metabolism following bone marrow transplantation. Bone. 26, 275, 2000.

45. Wang, H., Li, Y., Zuo, Y., Li, J., Ma, S., and Cheng, L. Biocompatibility and osteogenesis of biomimetic nanohydroxyapatite/polyamide composite scaffolds for bone tissue engineering. Biomaterials. 28, 3338, 2007.

46. Harris, S.A., Enger, R.J., Riggs, B.L., and Spelsberg, T.C. Development and characterization of a conditionally immor- talized human fetal osteoblastic cell line. J Bone Miner Res. 10, 178, 1995.

Address reprint requests to: Nicholas Kotov, Ph.D. 2300 Hayward Street 3074 H.H. Dow Building Ann Arbor, MI 48109

E-mail: kotov@umich.edu

Received: May 19, 2007 Accepted: February 20, 2008 
